\title{
The Osteogenic Capacity of Human Amniotic Membrane Mesenchymal Stem Cell (hAMSC) and Potential for Application in Maxillofacial Bone Reconstruction in Vitro Study
}

\author{
David B. Kamadjaja ${ }^{1,2 *}$, Purwati2,3,4, Fedik A. Rantam ${ }^{2,3}$, Ferdiansyah ${ }^{3,5}$, Coen Pramono ${ }^{1}$ \\ ${ }^{1}$ Department of Oral \& Maxillofacial Surgery, Faculty of Dentistry, Airlangga University, Surabaya, Indonesia \\ ${ }^{2}$ Laboratory of Stem Cell, Institute of Tropical Disease, Airlangga University, Surabaya, Indonesia \\ ${ }^{3}$ Regenerative Medicine \& Stem Cell Centre, Airlangga University \& Dr. Soetomo General Hospital, Surabaya, \\ Indonesia \\ ${ }^{4}$ Department of Internal Medicine, Dr. Soetomo General Hospital, Surabaya, Indonesia \\ ${ }^{5}$ Department of Orthopaedic \& Traumatology, Dr. Soetomo General Hospital, Surabaya, Indonesia \\ Email: ${ }^{*}$ davidbk65@gmail.com
}

Received 10 April 2014; revised 24 May 2014; accepted 7 June 2014

Copyright (C) 2014 by authors and Scientific Research Publishing Inc.

This work is licensed under the Creative Commons Attribution International License (CC BY).

http://creativecommons.org/licenses/by/4.0/

(c) (i) Open Access

\section{Abstract}

Amniotic membrane of human placenta is a source of abundant mesenchymal stem cell (hAMSC) which makes it a potential source of allogeneic multipotent cell for bone healing. However, much has to be explored about its isolation procedure and the osteogenic differentiation potential. The aims of this study are to establish the procurement procedure of human amniotic membrane, the isolation and culture of hAMSC, the MSC phenotypic characterization, and the in vitro osteogenic differentiation of hAMSC. Results of the study are as follows. The quality of human amniotic membrane would be best if procured from Caesarean operation under highly aseptic condition to avoid fungal and bacterial contamination on the culture. Isolation procedure using modified Soncini protocol yielded large amount of MSC with high proliferative capacity in culture medium. Characterization of hAMSC showed that the majority of the target cells exhibited specific MSC markers (CD105 and CD90) with a small number of these cells expressing CD45, the marker of hematopoeitic cells. The in vitro osteogenic differentiation of hAMSC followed by Alizarin Red staining showed that osteoblastic differentiation was detected in a significantly high number of cells. This study concludes that hAMSCs isolated from human amniotic membrane have the capacity for in vitro osteogenesis which makes them be one of the potential allogeneic stem cells for application in maxillofacial bone reconstruction.

${ }^{*}$ Corresponding author.

How to cite this paper: Kamadjaja, D.B., et al. (2014) The Osteogenic Capacity of Human Amniotic Membrane Mesenchymal Stem Cell (hAMSC) and Potential for Application in Maxillofacial Bone Reconstruction in Vitro Study. J. Biomedical Science and Engineering, 7, 497-503. http://dx.doi.org/10.4236/jbise.2014.78051 


\section{Keywords}

\section{Human Amniotic Membrane Mesenchymal Stem Cells, Modified Soncini Protocol, MSC Phenotypic Characterization, Osteogenic Differentiation, Allogeneic Stem Cell, Maxillofacial Bone Reconstruction}

\section{Introduction}

Large segmental defects in the mandible resulted from tumor resection do not regenerate spontaneously and normally. Therefore, it requires the jaw reconstruction to retain masticatory and speech function and facial esthetic. To date, gold standard of mandibular reconstruction is autogenous bone grafting because it is considered to be osteogenic, osteoconductive and osteoinductive [1]. However, autogenous bone graft has disadvantages, such as limited amount and shape of the donor graft available and the morbidity associated with complication of the donor site operation. It is desirable, therefore, to search for alternative means of bone grafting which do not cause donor site morbidity, has the unlimited availability and possesses the equal bone regeneration capacity as that of autogenous bone graft.

The fast development of tissue engineering has offered hope to obtain such an ideal bone replacement method. It combines three components i.e. multipotent cells, scaffold and growth factors. Mesencymal stem cells (MSC) are the most potential adult stem cell for bone regeneration due to their multipotential properties [2]. MSC can be obtained from various tissues in the body such as bone marrow, periosteum, peripheral blood, adipose tissues, and skin. The most widely used MSC for bone regeneration is bone marrow-derived MSC, also called BM-MSC because of its high osteogenic capacities compared to other sources of MSC [3]. However, BM-MSC has a few limitations. Bone marrow aspiration procedure is relatively invasive and may not yield quality stem cells if taken from elderly or medically compromised patients [4]. The challenge in tissue engineering today is, therefore, to look for other sources of MSC which provide a large number of stem cells and pose the minimal morbidity to the patients who need them.

Amniotic membrane of the human placenta is a source of abundant stem cells which consist of epithelial and stromal cell. Recent studies have shown that stromal cells of the human amniotic membrane, also called human amniotic mesenchymal stem cells (hAMSC), possess the similar characteristics as those of bone marrow mesenchymal stem cell. It was proven that hAMSC is capable of differentiating into three forms of germ layers and has anti-inflammatory effects and the low immunogenicity [5]-[9].

Human amniotic membrane-derived stem cell, in terms of its clinical application, has advantages over autogenous bone marrow-derived stem cell because no morbidity is involved in its procurement procedures, the unlimited amount of stem cells available [10]. Besides, the quality of autogenous BM-MSC in most cases is influenced by the patients' age and health condition. Isolation of hAMSC does not sacrifice embryo as it is the case with embryonic stem cells. Therefore, it does not cause any legal and ethical issues [11]. However, there has been no in vivo studies suggesting the potential clinical application of hASMC in maxillofacial bone tissue engineering. In order to support such in vivo experiments, an effective hAMSC isolation and cell expansion culture method should be established and its bone forming potential should be confirmed in vitro.

In this study, we try to establish 1) the procurement procedure of human amniotic membrane; 2) the isolation and culture of hAMSC; 3) the MSC phenotypic characterization; and 4) the in vitro osteogenic differentiation potential of hAMSC.

\section{Material and Methods}

\subsection{Procurement of Human Amniotic Membrane}

The human amniotic membrane was harvested from both per vaginam delivery and Cesarean section performed at the Central Operating Theatre Dr Soetomo General Hospital Surabaya. The procedure was legally and ethically approved by Committee for Ethics on Health Researches, Dr. Soetomo General Hospital. The fresh amnion was mechanically peeled from chorion, washed in phosphate-buffered saline (PBS) three times to remove the excess blood, and soaked in Ringer Lactate solution containing gentamycin and amphotericin B. 


\subsection{Isolation and Culture of hAMSCs}

The procedure for isolation and culture of hAMSC were performed at Laboratory of Stem Cell, Institute of Tropical Disease, Airlangga University, Surabaya. The isolation method was performed using modified Soncini's protocol (Prado et al., 2011). The amniotic membrane was minced up with knife into fine pieces and subsequently subjected to $0.25 \%$ trypsin for digestion to remove the epithelial cells, and after centrifugation the supernatant was removed away. This step was repeated twice. The crushed tissue was subsequently subjected to PBS containing $0.75 \mathrm{mg} / \mathrm{ml}$ collagenase IV (Sigma-Aldrich, St. Louis, MO, USA) and $0.075 \mathrm{mg} / \mathrm{mL}$ Dnase I (Takara Bio, Shiga, Japan), incubated at $37^{\circ} \mathrm{C}$ for 60 minutes. After filtration with cell strainer and pellet collection upon centrifugation for 5 minutes the cells were finally obtained

The single cells collected were then cultured on collagen-coated dishes using Dulbecco's minimal essential medium (DMEM)/F12 (1:1) (Gibco BRL, Gaithersburg, MD, USA), supplemented with human leukemia inhibitory factor (10 ng/mL) and fetal bovine serum (Gibco BRL). The medium was changed every three days and when the confluence reached $80 \%$ the cell splitting was done using trypsin. Half to two thirds of the cells were then replated with the same medium onto new dish.

\subsection{Phenotypic Characterization of hAMSC}

\subsubsection{Immunocytochemistry}

The cultured cells were plated onto coverslips and, after incubated at $37^{\circ} \mathrm{C}$ for 1 - 2 hours, fixed with formaldehyde $10 \%$ for 15 minutes. The coverslips were then rinsed four times with PBS and let it dry for few minutes. The cells were blocked with PBS and FBS 1\% for 15 - 30 minutes and then washed with PBS four times. FITC-labelled monoclonal antibody anti-human CD 105 and CD 45 was applied to the cells and incubated for 60 minutes. Thereafter, the cells were rinsed with PBS twice and they were ready for analysis using fluoresence microscope.

\subsubsection{Flow Cytometry}

Trypsinized MSC were resuspended in DMEM, washed with PBS, and fixed in formaldehyde $10 \%$ for 10 minutes, and finally closed in 10\% BSA for one hour. The cells were incubated with primary antibodies mouse anti-human CD90 and anti-human CD45 for 40 minutes. The unbound antibodies were removed by washing with PBS. FITC-conjugated anti-mouse antibody were used for labelling of the bound primary antibodies. After washing with PBS, the cells were analyzed using FACSCalibur flow cytometer (BD Biosciences, Franklin Lakes, NJ, USA).

\subsubsection{In Vitro Osteogenic Differentiation Studies}

hAMSC from passages 4 were detached using $2 \times$ trypsin solution and seeded into 24-well microplate containing osteogenic medium, which composed of DMEM/F12 medium supplemented with ascorbic acid, glycerolphosphate and dexamethasone, for osteogenic study. The same cells were also seeded into the same petri dish containing normal medium (DMEM/F12) for control. Both of the medium were changed every 2 to 3 days. The cells were cultured in the osteogenic medium for 21 days to induce the osteoblastic differentiation. After 21 days, the cells in the osteogenic medium as well as normal medium were detached from the petri dish and seeded into 24-well plate containing $10 \%$ formaldehyde for cell fixation. To evaluate osteogenesis, the presence of calcium deposits in the cultures was determined using Alizarin red stain according to a standard protocol.

\section{Results}

\subsection{Procurement of Human Amniotic Membrane}

The procurement of human amniotic membrane from normal, per vaginam, delivery procedure were done twice under aspetic condition, both of which resulted in the cell culture being contaminated with fungus. The amniotic membrane procurement with Cesarean section under the same aseptic condition yielded growth of cell cultures which were not contaminated.

The procurement procedure with Cesarean section was as follows. After the baby had been delivered through Caesarean section and umbilicard cord cut off, the placenta together with the remaining umbilicard cord was evacuated and placed in the sterile kidney-shape stainless steel receptacle. The amniotic membrane was then 
peeled off the underlying tissue which was chorion and cut out from the placenta. The membrane was then washed by immersing it repeatedly in two bottles containing PBS to remove blood and finally soaked in the third bottle containing medium DMEM/F12 supplemented with antibiotic and antifungal agents.

\subsection{Isolation and Culture of hAMSC}

The growth of the cells were observed for the first 24 hours, at which time the cells were mostly round or oval. After 24 hours, the majority of the cells were adherent to the plate and became spindle in shape. After 3 days, the cells had grown to a confluence of $90 \%$ and arranged in swirling pattern (Figure 1). The cell splitting procedure in this study was performed fourteen times before the cells were used for subsequent examination.

\subsection{Phenotypic Characterization of hAMSCs}

The immunocytochemistry showed that the target cells expressed strong mesenchymal stem cell marker CD 105 while weak expression of CD 45 was noticed (Figure 2). The result of flowcytometry showed that majority $(4.219,82)$ of the target cells expressed strong CD90, while CD45 was expressed in small number $(1.117,05)$ of cells (Figure 2).

\subsection{In Vitro Osteogenic Differentiation of hAMSC}

After 21 days, majority of the cells in osteogenic medium were stained bright red with Alizarin Red while only small quantity of cells in normal medium stained positively with Alizarin Red (Figure 3).

\section{Discussion}

Amniotic membrane procurement from placenta of per vaginam delivery, although much easier to perform, is not suggested concerning the high incidence of fungal contamination of the sample collected. In the authors' experience the procurement procedure should be done under highly aseptic condition as even a slight contamination of the sample, such as in normal delivery, may finally cause fungal overgrowing the cell culture.

Two isolation protocol for amniotic membrane stem cell exist in the literature, i.e. Alviano et al. and Soncini et al. The isolation procedure used in this study was that of Soncini et al. with slight modification. The choice for this protocol was based on the result of one study which showed that compared to Alviano protocol, Soncini's could produce much higher amount of stem cell in relatively short period of time [12].

The isolated cells exhibited spindle-shaped fibroblast-like cells which adhered to the petri dish and when cell confluence was achieved the cell colonies acquired a swirling pattern. This feature was consistent with the characteristic morphology of mesenchymal stem cell demonstrated by many studies in the literatures [13].

Immunocytochemistry and flowcytometry on the target cells showed weak expression of CD45, the specific phenotypic markers of hematopoeitic stem cell. This result was consistent with one study which showed that hAMSC isolated with Soncini protocol expressed CD117, surface markers of hematopoeitic cells, in $22.79 \%$ of the cell population [12]. It was assumed that the isolation procedure used in this study may have given such
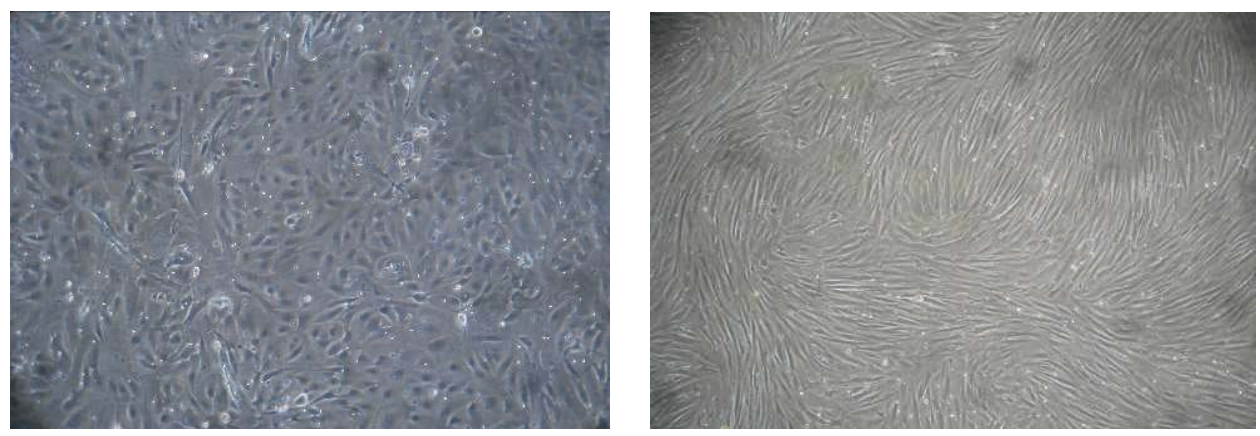

Figure 1. Culture of human amniotic mesenchymal stem cells (hAMSCs). On the first 24 hours the cells were mostly round or oval in shape (left); after 3 days the majority of the cells were adherent to the plate, showing spindle-shape or fibroblast-like cell morphology with swirling-pattern colonies, and has reached conluence (right) (inverted microscope, 200× magnification). 

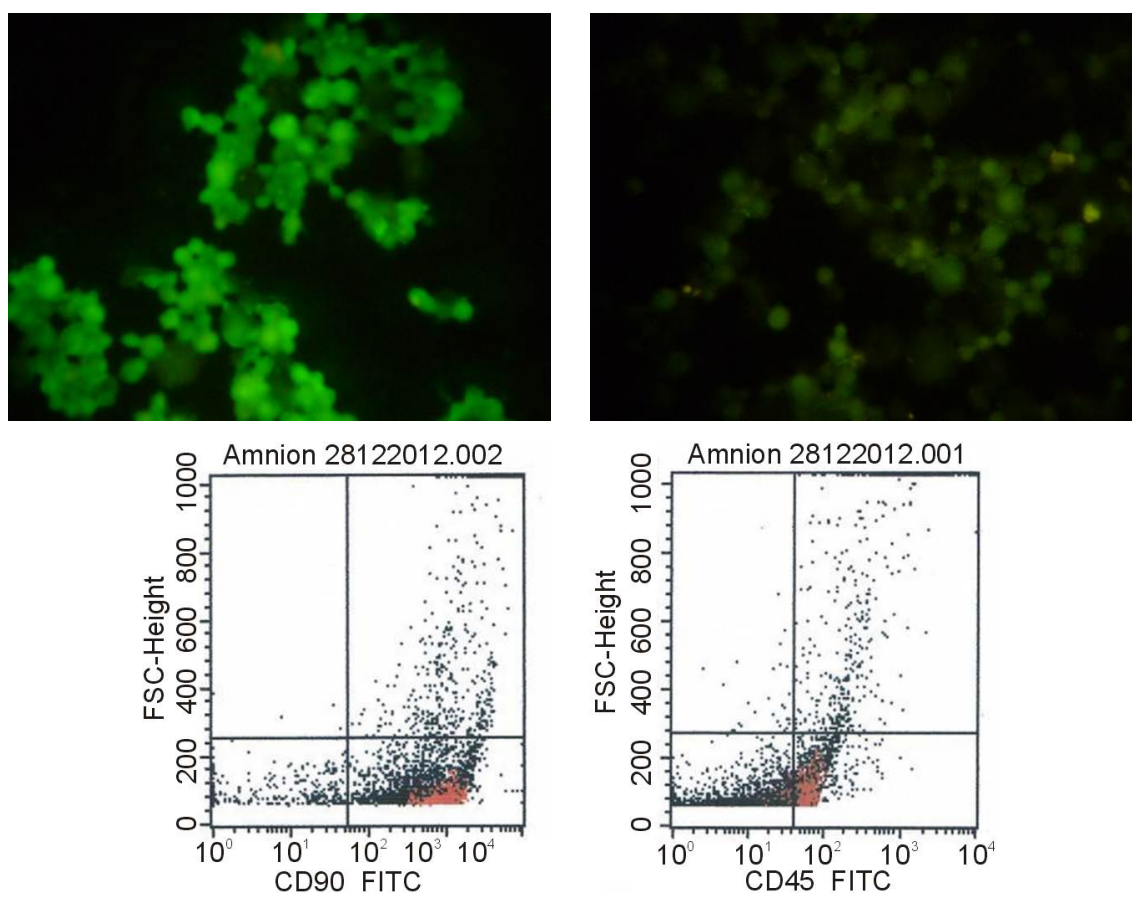

\begin{tabular}{cc}
\hline Markers & mean of $(+)$ ve expressions \\
\hline CD90 & 4.21982 \\
CD45 & 1.11705 \\
\hline
\end{tabular}

Figure 2. Phenotypic characterization of hAMSC. The immunocytochemistry result showed that the cells strongly expressed CD105 (top left) and weakly expressed CD45 (top right); Flowcytometry result showed that the collected cells showed strong expression for CD 90 but weak for CD 45 (middle \& bottom).
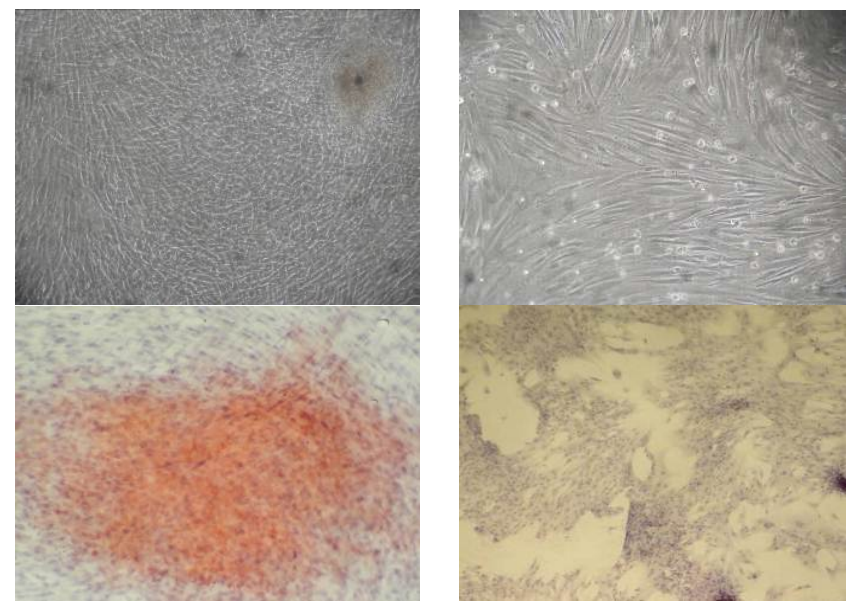

Figure 3. Microscopic feature of osteogenically-induced cell culture before and after staining with Alizarin Red. hAMSC culture in osteogenic medium after 21 days showing morphology of round or oval-shaped cells (upper left) and in normal medium the cells showing features of MSC (upper right). Upon staining with Alizarin Red the osteogenic medium exhibited large clusters with bright red appearance indicating the presence of extracellular mineral deposit (lower left) while the normal medium showed no color changes detected (lower right). (Alizarin Red staining, counter-stained with Mayer's hematoxylin, 200× magnification). 
condition that the cell culture may not be purely those of isolated mesenchymal stem cells, in other words, the isolation protocol had not completely separated the hematopoeitic stem cells from the MSC colonies.

One of the simple methods to identify osteoblastic differentiation in cell cultures was detecting the presence of extracellular calcium deposits. Calcium deposits can be specifically stained with Alizarin Red in which osteoblasts appear bright red in colour, while undifferentiated MSCs do not exhibit the colour [14]. The osteogenic-induced culture in this study showed large amount of positively-stained cell cluster indicating that hAMSC collected in this study differentiated towards osteoblast lineage when osteogenically induced by their environment. This was in accordance with the results of other studies in which cells isolated from amniotic membrane showed the characteristic features of mesenchymal stromal cells consistently exhibiting multilineage differentiation capacities towards bone, cartilage, and adipose tissue [15] [16].

It was quite surprising to note that the osteogenic-induced cultures in this study did not exhibit positive staining in all obseved areas as reported by other studies. This could be attributed to the use of cells with high passage numbers. The cells used in this osteogenic differentiation study were from fourteenth passage. It is recommended that cell with low passage number, less than 5 passsages, be used in such studies, as mesenchymal stem cells tend to lose their diffferentiation potential with increasing passage number. Use of cells with high passage number cell might lead to false-positive or false-negative results. It was also suggested that for such purposes, cells be subcultured when they are $70 \%$ - $80 \%$ confluent, as $100 \%$ confluence would result in loss of multipotency of the cells [4].

\section{Conclusion}

Based on the results of this study, it was concluded that MSC collected from human amniotic membrane had the capacity for in vitro osteogenesis which make them be the one of the potential allogeneic mesenchymal stem cells for bone tissue engineering. However, further studies should be done to explore its application potential in maxillofacial bone reconstruction.

\section{Acknowledgements}

This work was supported by grants from DIPA BOPTN Fiscal Year 2013.

\section{References}

[1] Randall, M.W. (2004) Bony Reconstruction of the Jaws. In: Miloro, M., Ed., Peterson's Principles of Oral and Maxillofacial Surgery, 2nd Edition, BC Decker Inc., London, 783.

[2] Caplan, A.I. (2007) Fundamental of Stem Cells Tissue Engineering. In: Fisher, J.P., Mikos, A.G. and Bronzino, J.D., Eds., Tissue Engineering, CRC Press, Boca Raton, 1-1, 1-9.

[3] Sakaguchi, Y., Sekiya, I., Yagishita, K. and Muneta, T. (2005) Comparison of Human Stem Cells Derived from Various Mesenchymal Tissues. Superiority of synovium as a cell source. Arthritis \& Rheumatism, 52, 2521-2529. http://dx.doi.org/10.1002/art.21212

[4] Kretlow, J.D., Yu, Q.J., Wei, L., Wen, J.Z., Tan, H.H., Zhou, G.D., Baggett, L.S., Mikos, A.G. and Cao, Y.L. (2008) Donor Age and Cell Passage Affects Differentiation Potential of Murine Bone Marrow-Derived Stem Cells. BMC Cell Biology, 9, 60. http://dx.doi.org/10.1186/1471-2121-9-60

[5] Tseng, S.C.G., Li, D.Q. and Ma, X. (1999) Suppression of Transforming Growth Factor-Beta Isoforms, TGF-Receptor Type II, and Myofibroblast Differentiation in Cultured Human Corneal and Limbal Fibroblasts by Amniotic Membrane Matrix. Journal of Cellular Physiology, 179, 325-335. http://dx.doi.org/10.1186/1471-2121-9-60

[6] Miki, T., Lehmann, T., Cai, H., Stolz, D.B. and Strom, S.C. (2005) Stem Cell Characteristics of Amniotic Epithelial Cells. Stem Cells, 23, 1549-1559. http://dx.doi.org/10.1634/stemcells.2004-0357

[7] Solomon, A., Rosenblatt, M., Monroy, D., Ji, Z., Pflugfelder, S.C., Tseng, S.C.G. (2001) Suppression of Interleukin 1 (Alpha) and Interleukin 1 (Beta) in Human Limbal Epithelial Cells Cultured on the Amniotic Membrane Stromal Matrix. British Journal of Ophthalmology, 85, 444-449. http://dx.doi.org/10.1136/bjo.85.4.444

[8] Kubo, M., Sonoda, Y., Muramatsu, R. and Usui, M. (2001) Immunogenicity of Human Amniotic Membrane in Experimental Xenotransplantation. Investigative Ophthalmology \& Visual Science, 42, 1539-1546.

[9] Wang, M., Yoshida, A., Kawashima, H., Ishizaki, M., Takahashi, H. and Hori, J. (2006) Immunogenicity and Antigenicity of Allogeneic Amniotic Epithelial Transplants Grafted to the Cornea, Conjunctiva, and Anterior Chamber. Investigative Ophthalmology \& Visual Science, 47, 1522-1532. http://dx.doi.org/10.1167/iovs.05-0787 
[10] Ilancheran, S., Moodley, Y. and Manuelpillai, U (2009) Human Fetal Membranes: A Source of Stem Cells for Tissue Regeneration and Repair? Placenta, 30, 2-10. http://dx.doi.org/10.1016/j.placenta.2008.09.009

[11] Toda, A., Okabe, M., Yoshida, T. and Nikaido, T. (2007) The Potential of Amniotic Membrane/Amnion-Derived Cells for Regeneration of Various Tissues. Critical review. Journal of Pharmacological Sciences, 105, 215-228. http://dx.doi.org/10.1254/jphs.CR0070034

[12] Diaz-Prado, S., Lopez, E.M., Gomez, T.H., Vazquez, E.R., Boquete, I.F., de Toro, F.J. and Blanco, F.J. (2011) Isolation and Characterization of Mesenchymal Stem Cells from Human Amniotic Membrane. Tissue Engineering Part C, 17, 49-59. http://dx.doi.org/10.1089/ten.tec.2010.0136

[13] Shuang, Z.H., Ping, S. and Xi, N.P. (2010) Culture and Identification of Human Amniotic Mesenchymal Stem Cells. Original article. Chinese Medical Sciences Journal., 25, 211-214. http://dx.doi.org/10.1016/S1001-9294(11)60004-7

[14] Gregory, C.A., Gunn, W.G., Peister, A. and Prockop, D.J. (2005) An Alizarin Redbased Assay of Mineralization by Adherent Cells in Culture: Comparison with Cetylpyridinium Chloride Extraction. Analytical Biochemistry, 329, 77-84. http://dx.doi.org/10.1016/j.ab.2004.02.002

[15] Pittenger, M.F., MacKay, A.M., Beck, S.C., et al. (1999) Multilineage Potential of Adult Human Mesenchymal Stem Cells. Science, 284, 143-147. http://dx.doi.org/10.1126/science.284.5411.143

[16] Jiang, Y., Jahagirdar, B.N. and Reinhardt, L.R. (2002) Pluripotency of Mesenchymal Stem Cells Derived from Adult Marrow. Nature, 418, 41-49. http://dx.doi.org/10.1126/science.284.5411.143 
Scientific Research Publishing (SCIRP) is one of the largest Open Access journal publishers. It is currently publishing more than 200 open access, online, peer-reviewed journals covering a wide range of academic disciplines. SCIRP serves the worldwide academic communities and contributes to the progress and application of science with its publication.

Other selected journals from SCIRP are listed as below. Submit your manuscript to us via either submit@scirp.org or Online Submission Portal.
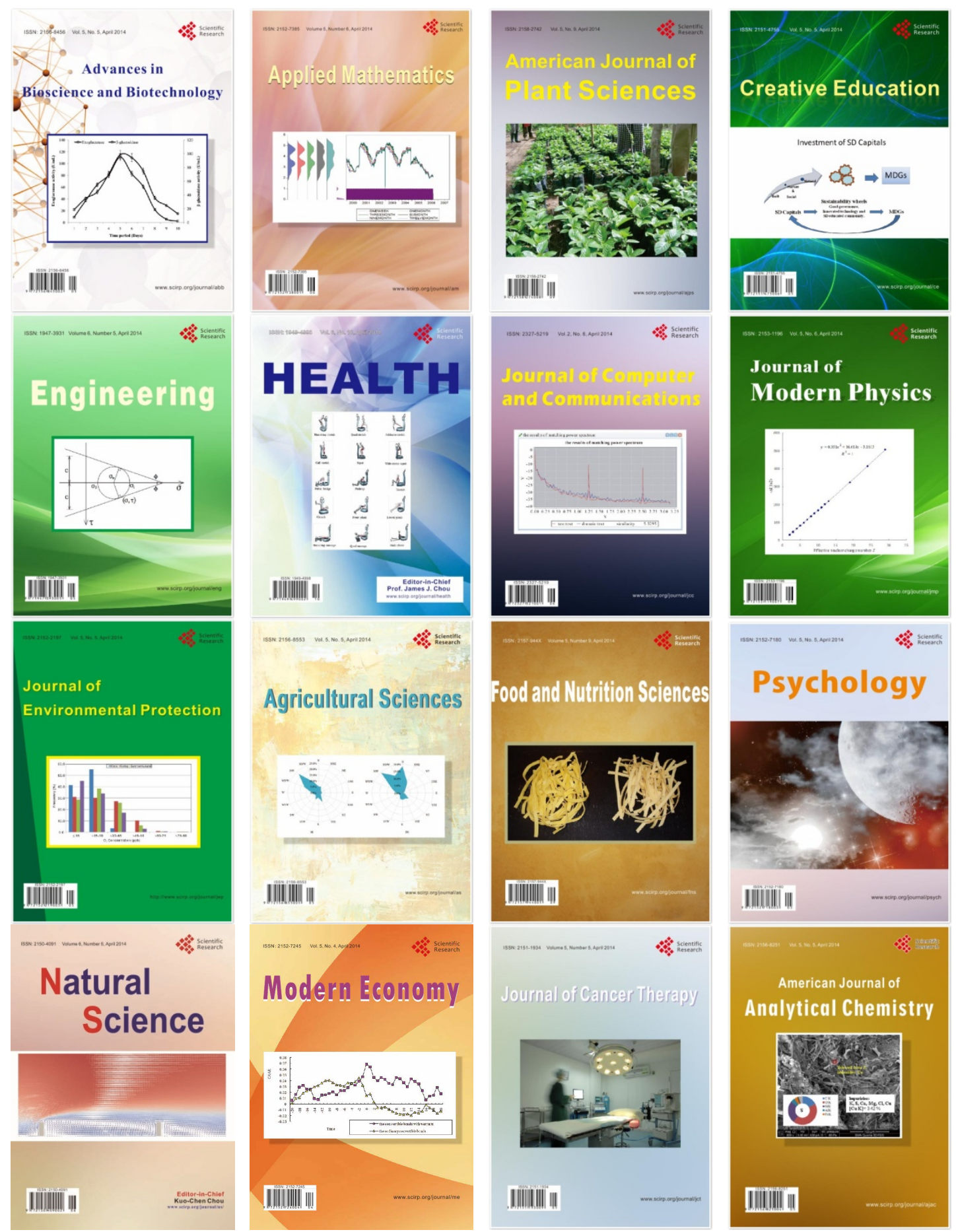